\title{
Dipolar Triplet States of $p$-Nitroaniline and $N$-Alkyl Derivatives with One-, Two-, and Three-Fold Symmetry
}

\author{
Wouter Schuddeboom and John M. Warman* \\ IRI, Delft University of Technology, Mekelweg 15, 2629 JB Delft, The Netherlands
}

\author{
H. A. M. Biemans and E. W. Meijer \\ Laboratory of Organic Chemistry, Eindhoven University, P.O. Box 513, 5600 MB Eindhoven, The Netherlands
}

Received: March 6, 1996; In Final Form: May 8, $1996^{\otimes}$

\begin{abstract}
Photoexcitation of $p$-nitroaniline (PNA) and its $N$-alkyl derivatives results in the formation of triplet states with dipole moments considerably larger than that of the ground state, as monitored by time-resolved microwave conductivity (TRMC). The triplet state lifetime, $\tau_{\mathrm{T}}$, is only $54 \mathrm{~ns}$ for PNA in benzene but increases to 1400 ns on $N, N$-dimethyl substitution and to $275 \mathrm{~ns}$ in the more polar solvent $p$-dioxane. This sensitivity of $\tau_{\mathrm{T}}$ is attributed to the close proximity of all of the lowest lying singlet and triplet $n \pi^{*}$ and $\pi \pi^{*}$ states of PNA, which results in substantial $\mathrm{S}_{1} \leftrightarrow \mathrm{T}_{1}$ mixing. The dipole moment of the "pure" ${ }^{3} \pi \pi^{*}$ state is estimated to be 11 D. Methylene-bridged arrays of PNA moieties with two- and three-fold $D_{n}$ symmetry, 2-PNA and 3-PNA, are also found to have highly dipolar triplet states, indicating that symmetry breaking and exciton localization occur in $T_{1}$. Flip-flop interchange between the resonant dipolar structures is estimated to take place on a time scale of tens of picoseconds. Collective interactions between the PNA moieties in the multichromphoric arrays results in a marked blue shift in the first absorption maximum from $384 \mathrm{~nm}$ for 1-PNA to $375 \mathrm{~nm}$ for 2-PNA and to $354 \mathrm{~nm}$ for 3-PNA.
\end{abstract}

\section{Introduction}

The photophysics of $p$-nitroaniline (PNA) and its $N$-alkyl derivatives have been extensively studied using a variety of optical detection techniques. ${ }^{1-11}$ A common feature found is an extremely short $S_{1}$ lifetime and very weak, if any, fluorescence. For PNA itself only phosphorescent emission has ever been observed. On $N$-methyl substitution weak fluorescence is found together with phosphorescence with the former increasing in relative intensity in more polar solvents. Studies of the excitation-wavelength dependence of the fluorescence to the phosphorescence ratio and the relative polarization of the emissions for $N$-methyl substituted PNA indicate the presence of two singlet state transitions within the absorption envelope. $^{7-11}$ On the basis of electrochromic effects on the absorption of PNA and its high efficiency in second harmonic generation (SHG), it can be concluded that vertical excitation within the first absorption band leads to a highly dipolar excited singlet state. The dipole moment has been estimated to be approximately 15 D. 2,3,12,13

Intersystem crossing is clearly an important radiationless decay channel for the $S_{1}$ state of PNA derivatives. Despite this, little quantitative data exist on the efficiency of intersystem crossing or the nature of the triplet state formed. The complete lack of fluorescence for PNA itself suggests that the intersystem crossing efficiency, $\phi_{\text {isc }}$, is probably close to unity. An estimate of 0.4 for $\phi_{\text {isc }}$ has been made for $N$-methyl-p-nitroaniline in a poly(methyl methacrylate) matrix using the time-resolved thermal lens method. ${ }^{10}$ In a flash-photolysis study of PNA in EPA solution an absorption spectrum with a maximum at 595 nm was observed at low temperatures and was assigned to a charge transfer triplet state. ${ }^{5}$ The lifetime of the $\mathrm{T}_{1}$ absorption decreased markedly with increasing temperature to be replaced by an absorption at $c a$. $480 \mathrm{~nm}$ which was attributed to a photochemical product.

${ }^{\otimes}$ Abstract published in Advance ACS Abstracts, July 1, 1996.
Interest in the optoelectric properties of PNA and related nitro-anilino compounds has increased in recent years because of their efficient nonlinear-optical properties, in particular in SHG. PNA itself is frequently used as a benchmark standard. Until recently SHG activity was thought to be restricted to conjugated donor-acceptor molecules with a non-centrosymmetric charge distribution such as PNA. The discovery by Ledoux et al. ${ }^{14}$ that the symmetrical relative of PNA, 1,3,5trinitro-2,4,6-triaminobenzene, displays a large SHG efficiency in the solid phase has however led to an upsurge of interest, from both the theoretical and the practical point of view, in the photophysical properties of symmetrical donor-acceptor compounds. ${ }^{15-18}$

In the present work we have investigated the photophysics of PNA and compounds containing symmetrical arrays of $N$-alkyl substituted PNA moieties, the structures of which are shown in Figure 1. The main method of investigation used was time-resolved microwave conductivity (TRMC) with which short-lived dipolar products of flash photolysis can be monitored. ${ }^{19,20}$

\section{Experimental Section}

The structures of the solute molecules studied in the present work are shown in Figure 1 together with the pseudonyms used in the text. PNA and DMPNA were obtained commercially (Eastman Kodak). The synthesis and purification of compounds 1-PNA, 2-PNA, and 3-PNA were performed according to published procedures. ${ }^{21-23}$ The molecular characterizations by ${ }^{1} \mathrm{H}$ - and ${ }^{13} \mathrm{C}-\mathrm{NMR}$, melting point determination, and elemental analysis were in agreement with published data. The solvents used were UV spectroscopic grade benzene and $p$-dioxane from Fluka. The latter was passed over a column of freshly activated silica gel immediately prior to use to remove traces of water.

The optical absorption spectra shown in Figure 2 were made using a UVIKON $940 \mathrm{UV} / \mathrm{vis}$ spectrophotometer. Timeresolved fluorescence measurements were carried out by flash 

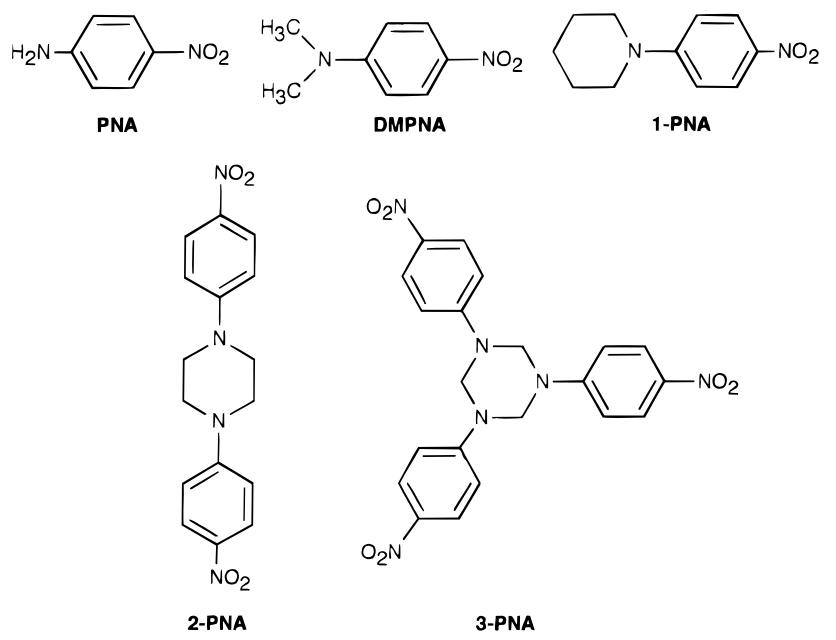

Figure 1. Molecular structures of the compounds studied in the present work together with the acronyms used in the text.
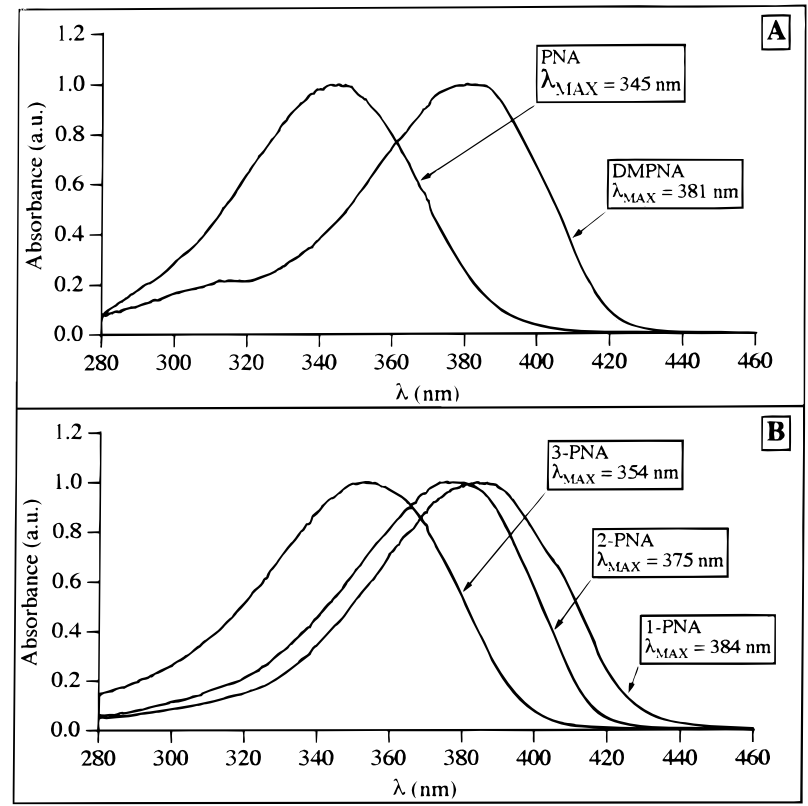

Figure 2. Optical absorption spectra of benzene solutions of the compounds shown in Figure 1, normalized to 1.0 at the wavelength of maximum absorption.

photolysis of $c a .0 .2 \mathrm{~cm}^{-1}$ absorbance solutions using a $0.8 \mathrm{~ns}$, $1.4 \mathrm{~mJ}, 337 \mathrm{~nm}$ pulse from a PRA LN1000 $\mathrm{N}_{2}$ laser. After passage through a Jobin-Yvon monochromator, the emitted light was detected using an ITL TF 1850, 100 ps rise time photodiode, the output of which was fed, after amplification, to a 7912AD Tektronix digitizer. Decay times longer than 200 ps could be measured.

TRMC experiments were carried out using a Lumonics HyperEX 400 excimer laser (7 ns, $50 \mathrm{~mJ}, 308 \mathrm{~nm}$ pulse). The solutions of absorbance $0.5-2.0 \mathrm{~cm}^{-1}$ were deaerated by purging with $\mathrm{CO}_{2}$ gas. The TRMC method of detecting changes in the dipole moment of solute molecules on photoexcitation has been described fully elsewhere. ${ }^{19,20}$ The RC response time of detection was controlled mainly by the microwave cavity characteristics. This was accurately known and was close to 5 ns. TRMC transients are shown on a time scale of $0-80 \mathrm{~ns}$ in Figures 3 and 4 for all solutions studied. Longer time scales, up to $10 \mu \mathrm{s}$, were also monitored and used to determine the transient decay times. The dotted kinetic fits to the data in Figures 3 and 4 were obtained by numerical solution of the differential equations describing the formation and decay of the

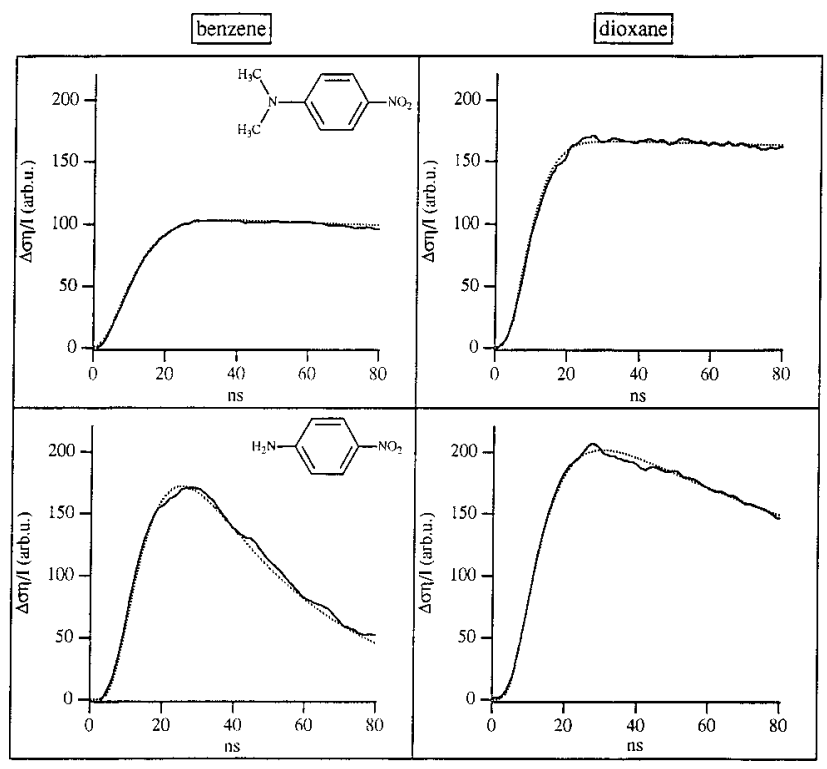

Figure 3. Transient increases in the microwave conductivity (dielectric loss) on flash photolysis at $308 \mathrm{~nm}$ of benzene and dioxane solutions of DMPNA and PNA.

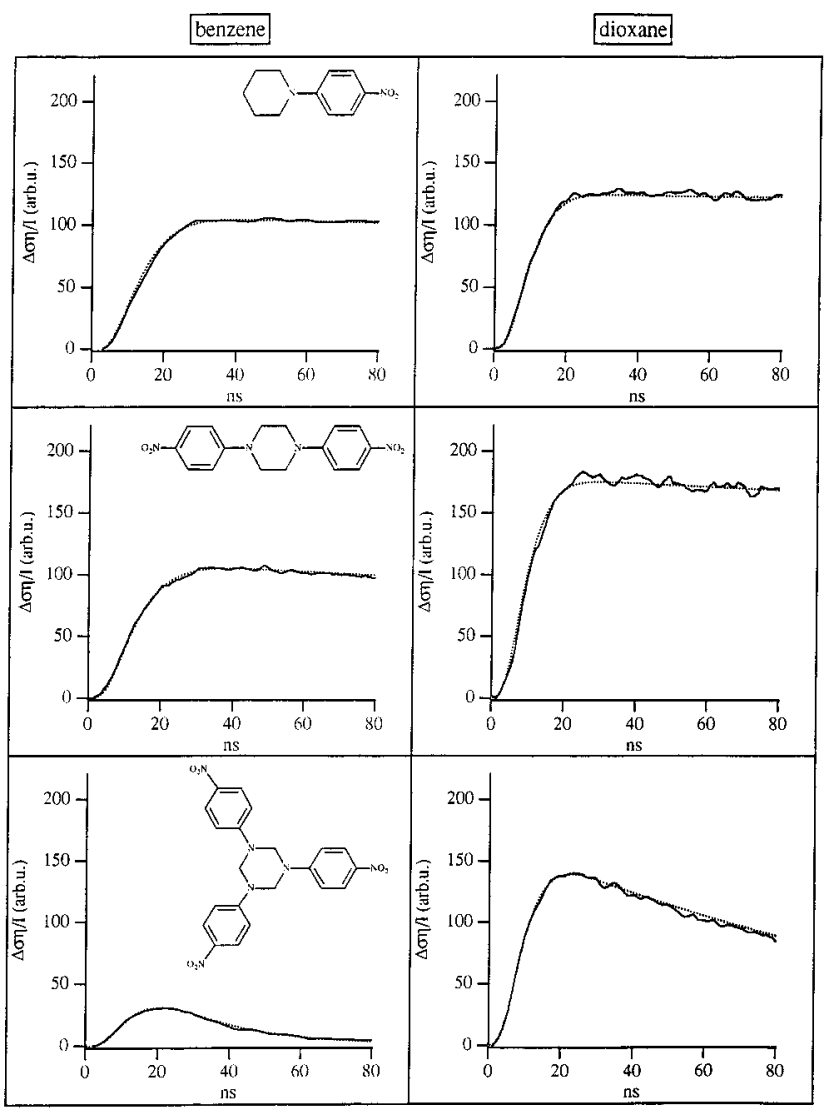

Figure 4. Transient increases in the microwave conductivity (dielectric loss) on flash photolysis at $308 \mathrm{~nm}$ of benzene and dioxane solutions of 1-, 2-, and 3-PNA.

triplet state, including convolution over the laser pulse shape and the cavity $\mathrm{RC}$ response function. ${ }^{19}$

Compound 2-PNA was found to be particularly sensitive to photodegradation of the TRMC signal, and care had to be taken to use as few single pulses as possible. After sustained irradiation of 2-PNA in benzene a new absorption band at longerwavelengths $\left(\lambda_{\max }=c a .460 \mathrm{~nm}\right)$ was found. No indication of photodegradation was found for the other compounds investigated. 
TABLE 1: Wavelength/Energy Maximum and Half-Width of the First Optical Absorption Band and the Wavelength Maximum and Quantum Yield of Fluorescence of the Compounds Shown in Figure 1 in Benzene Solution

\begin{tabular}{|c|c|c|c|c|c|}
\hline \multirow[b]{2}{*}{ Solute } & \multicolumn{3}{|c|}{ absorption } & \multicolumn{2}{|c|}{ emission $^{a}$} \\
\hline & $\lambda_{\mathrm{A}}(\mathrm{nm})$ & $h v_{\mathrm{A}}(\mathrm{eV})$ & $\Delta h v_{\mathrm{A}}(\mathrm{eV})$ & $\lambda_{\mathrm{F}}(\mathrm{nm})$ & $\phi_{\mathrm{F}}$ \\
\hline PNA & 345 & 3.59 & 0.63 & & $\ll 10^{-3}$ \\
\hline DMPNA & 381 & 3.25 & 0.51 & $430 \pm 10$ & $\sim 10^{-3}$ \\
\hline 1-PNA & 384 & 3.23 & 0.54 & $430 \pm 10$ & $\sim 10^{-3}$ \\
\hline 2-PNA & 375 & 3.31 & 0.55 & & $<10^{-3}$ \\
\hline 3-PNA & 354 & 3.50 & 0.64 & & $\ll 10^{-3}$ \\
\hline
\end{tabular}

${ }^{a}$ In all cases the fluorescence lifetime, $\tau_{\mathrm{F}}$, was less than the time resolution of detection, i.e., $<200$ ps.

\section{Results and Discussion}

Optical Absorption. The broad, unstructured first absorption bands of the compounds studied are shown in Figure 2. The absorption maxima and full widths at half-maximum (fwhm) are listed in Table 1. The pronounced bathochromic shift on $N$-alkyl substitution, illustrated by PNA and its $N, N$-dimethyl derivative DMPNA, is characteristic of aniline derivatives and is a consequence of the increased electron density at the anilino nitrogen caused by the electron-donating effect of the alkyl groups. The shift in $\lambda_{\max }$ from $345 \mathrm{~nm}(3.59 \mathrm{eV})$ for PNA to $381 \mathrm{~nm}(3.25 \mathrm{eV})$ for DMPNA increases by a further $3 \mathrm{~nm}$ on substitution of the pentamethylene ring at the anilino nitrogen to give 1-PNA.

Studies of the wavelength dependence of the polarization and relative yield of fluorescence and phosphorescence have clearly demonstrated that the first absorption band of PNA derivatives involves transitions to more than one electronic configuration. 4,6,8,9,11 Evidence of slight shoulders on the long-wavelength side of the absorption spectra of DMPNA and 1-PNA are apparent and are probably due to an increased separation between these lowest lying excited singlet levels. Despite this, the overall half-widths are less than for PNA itself. The larger half-width for PNA may in some way be due to the strong $S_{1}$ $\leftrightarrow \mathrm{T}_{1}$ mixing which occurs and which is considered to be responsible for the very short triplet state lifetime discussed below.

The absorption spectra of the molecules consisting of symmetrical, methylene-bridged arrays of two and three PNA moieties, 2-PNA and 3-PNA, are very similar in shape to those containing only a single PNA unit. No additional longwavelength absorption bands indicative of intramolecular electron exchange interactions between the PNA chromophoric units are apparent. The spectra suggest rather that, due to the decoupling effect of the intervening methylene groups, photon absorption involves local excitation of a single PNA moiety.

The absorption maximum undergoes a pronounced shift to the blue in going from 1-PNA $(384 \mathrm{~nm} ; 3.23 \mathrm{eV})$ to 2-PNA $(375 \mathrm{~nm} ; 3.31 \mathrm{eV})$ to 3 -PNA $(354 \mathrm{~nm} ; 3.50 \mathrm{eV})$. This hypsochromic shift is ascribed to a mutual electron withdrawing effect of the anilino nitrogens in 2-PNA and 3-PNA. This counteracts and eventually almost neutralizes the electron donating effect of the intervening methylene groups and results in the spectrum for 3-PNA being similar in both wavelength maximum and half-width to that for the unsubstituted PNA parent compound. This effect results in a decrease in absorption at longer wavelengths which is a potential advantage in the generation of blue light by SHG. The large decrease in absorption in the $400-420 \mathrm{~nm}$ region in going from 1-PNA to 3 -PNA can be seen in Figure 2.

Optical Emission. In agreement with previous observations, ${ }^{9}$ no fluorescence at all could be detected from benzene solutions
TABLE 2: Triplet State Lifetimes and [ $\left.\phi_{\text {isc }} \Delta M_{\mathrm{T}}\right]$ Values Derived from TRMC Transients

\begin{tabular}{lccccc}
\hline & \multicolumn{2}{c}{$\tau_{\mathrm{T}}(\mathrm{ns})$} & & {$\left[\phi_{\mathrm{isc}} \Delta M_{\mathrm{T}}\right]\left(10^{-8} \mathrm{~m}^{2} /(\mathrm{V} \mathrm{s})\right)$} \\
\cline { 2 - 3 } \cline { 5 - 6 } solute & benzene & dioxane & & benzene & dioxane \\
\hline PNA & $54^{a}$ & $275^{a}$ & & 2.69 & 0.66 \\
DMPNA & 1400 & 2200 & & 1.09 & 0.56 \\
1-PNA & 1400 & 2600 & & 0.92 & 0.38 \\
2-PNA & 575 & 1200 & & 1.13 & 0.60 \\
3-PNA & 18 & 120 & & 0.90 & 0.53 \\
\multicolumn{2}{l}{${ }^{a}$ Extrapolated value at "zero concentration". }
\end{tabular}

of PNA at room temperature. Weak emissions with maxima at approximately $430 \mathrm{~nm}$ were observed for the $N$-alkyl substituted compounds DMPNA and 1-PNA. The lifetimes of the fluorescent states were however shorter than the $200 \mathrm{ps}$ time resolution of detection, and the quantum yields were estimated to be only on the order of $10^{-3}$. For the symmetrical compound 2-PNA, a very weak fluorescence was just detectable. Compound 3-PNA displayed a complete lack of fluorescence as found for PNA itself. The emissive properties therefore show a trend similar to that found for the absorption spectra, i.e., a tendency for 2-PNA and 3-PNA to revert back to the properties of the unsubstituted parent compound.

The rapid, mainly radiationless decay of the $S_{1}$ state of $p$-nitroanilines is undoubtedly due, to a large extent, to very rapid intersystem crossing to the triplet manifold. This is demonstrated by the large yields of phosphorescence which are found in low-temperature rigid matrices. ${ }^{4,9}$ In the case of PNA itself, only phosphorescent emission has in fact ever been observed and the quantum yield for triplet state formation is most probably close to unity. For $N$-alkyl substituted derivatives both fluorescence and phosphorescence are found, ${ }^{4,9}$ indicating that internal conversion of $S_{1}$ to $S_{0}$ can now compete with intersystem crossing to $T_{1}$. Phosphorescence is however still found to be the dominant process at low temperatures, even for DMPNA for which the phosphorescence to fluorescence ratio has been determined to be approximately $2: 1{ }^{9}$

TRMC Measurements. Readily measurable changes in the microwave conductivity (dielectric loss) are found on flash photolysis of benzene and dioxane solutions of all of the present compounds, including the symmetrical derivatives, as shown in Figures 3 and 4. In light of the discussion in previous sections, the TRMC transients are assigned to the formation of triplet states of the solutes with dipole moments substantially larger than those of the ground state molecules. Before considering the absolute magnitudes of the transients and the dipole moments of the $T_{1}$ states, the decay kinetics will be presented and discussed.

Triplet State Lifetimes. The $\mathrm{T}_{1}$ lifetimes determined from the decay of the TRMC transients are listed in Table 2. The value of $54 \mathrm{~ns}$ for PNA in benzene ${ }^{21}$ is unusually short for a $\mathrm{T}_{1}$ to $\mathrm{S}_{0}$ transition. We ascribe this to substantial mixing between $T_{1}$ and $S_{1}$, resulting in considerable singlet character of $T_{1}$ and an enhanced efficiency for intersystem crossing to $S_{0}$. This strong $S_{1} \leftrightarrow T_{1}$ mixing indicates that $S_{1}$ and $T_{1}$ are close in energy and probably of different electronic configurations, i.e., ${ }^{1} \mathrm{n} \pi^{*}$ and ${ }^{3} \pi \pi^{*}$. This is consistent with the order of states proposed for PNA in nonpolar solvents by McGlynn et al. ${ }^{8}$

As mentioned previously, the lowest ${ }^{1} \mathrm{n} \pi^{*}$ and ${ }^{1} \pi \pi^{*}$ are also close in energy and both contribute to the first absorption band of PNA. ${ }^{4,8,9}$ The general conclusion is therefore that all of the lowest $\mathrm{n} \pi^{*}$ and $\pi \pi^{*}$ singlet and triplet states of PNA are in fact very close to each other.

Alkyl substitution at the amino nitrogen or increased solvent polarity should lower the more polar $\pi \pi^{*}$ states relative to $\mathrm{n} \pi^{*}$, 


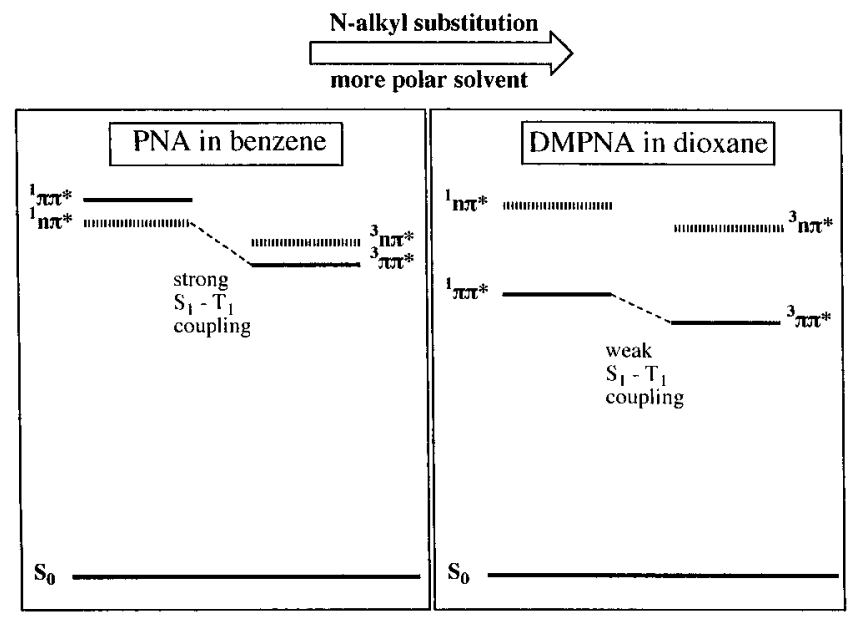

Figure 5. Order of states required to explain the short $T_{1}$ lifetime of PNA in benzene and the pronounced increase in lifetime found on $\mathrm{N}$-methyl substitution or increasing solvent polarity due to the change in $S_{1}$ from mainly ${ }^{1} n \pi^{*}$ to ${ }^{1} \pi \pi^{*}$ character.

causing eventually inversion in $\mathrm{S}_{1}$ from ${ }^{1} \mathrm{n} \pi^{*}$ to ${ }^{1} \pi \pi^{*} .{ }^{8} \mathrm{~S}_{1}$ and $\mathrm{T}_{1}$ will then both be of $\pi \pi^{*}$ character, and the $\mathrm{S}_{1} \leftrightarrow \mathrm{T}_{1}$ mixing responsible for the short $\mathrm{T}_{1}$ lifetime should decrease substantially. This explains the pronounced increase in the triplet state lifetime of PNA from 54 to $1400 \mathrm{~ns}$ on $N$-dimethyl substitution and to $275 \mathrm{~ns}$ on going from benzene to the more polar solvent dioxane. The decreased coupling between $S_{1}$ and $T_{1}$ also explains the occurrence of $\mathrm{S}_{1} \rightarrow \mathrm{S}_{0}$ fluorescence for the $N$-alkyl derivatives DMPNA and 1-PNA, resulting as it does in a decrease in the rate of the competitive $S_{1} \rightarrow T_{1}$ radiationless pathway.

The triplet lifetimes for 1-, 2-, and 3-PNA display a similar trend to that found for the optical absorption and emission properties with the results for 2-PNA and 3-PNA tending to revert back to those of PNA itself. In the case of 3-PNA the $\mathrm{T}_{1}$ lifetime of $18 \mathrm{~ns}$ is in fact even shorter than that for PNA, indicating even stronger mixing between the $S_{1}$ and $T_{1}$ states for this compound.

Absolute TRMC Measurements. Using a fitting procedure which has been fully described previously, ${ }^{19,20}$ the parameter $\left[\phi \Delta M_{*}\right]$ can be derived from the absolute magnitude of the TRMC transients. $\phi$ is the quantum yield of a photolytic product, and $\Delta M_{*}$ is the difference between the rotational charge mobility of the product and that of the solute molecule from which it is formed, i.e.,

$$
\Delta M_{*}=M_{*}-M_{0}
$$

The rotational charge mobility of a molecule is related to its dipole moment, $\mu$, and the dipole relaxation time, $\theta$, by

$$
M=K \mu^{2} F(\omega \theta) / \theta
$$

with

$$
K=\left(\epsilon^{\prime}+2\right)^{2} / 27 e k_{\mathrm{B}} T
$$

In (2), $\epsilon^{\prime}$ is the dielectric constant of the solution, $e$ is the elementary charge, $k_{\mathrm{B}} T$ is the characteristic thermal energy, $\omega$ is the radian frequency of the microwaves, and $F(\omega \theta)$ is the dispersion term, which for a single exponential, Debye-type relaxation is given by

$$
F(\omega \theta)=(\omega \theta)^{2} /\left(1+(\omega \theta)^{2}\right)
$$

For triplet state formation the experimentally determined parameter $\left[\phi \Delta M_{*}\right]$ can be equated with $\left[\phi_{\text {isc }} \Delta M_{\mathrm{T}}\right]$, with $\phi_{\text {isc }}$ the quantum yield for intersystem crossing and $\Delta M_{\mathrm{T}}$ the difference between the dipole moments of the triplet state and the ground state. The values of $\left[\phi_{\text {isc }} \Delta M_{\mathrm{T}}\right]$ determined for the solutesolvent combinations studied here are listed in Table 2 .

If the dipole relaxation time of the triplet state is taken to be equal to that of the ground state, then from (1) and (2) one has

$$
\left[\phi_{\text {isc }} \Delta M_{\mathrm{T}}\right]=\phi_{\text {isc }} K\left(\mu_{\mathrm{T}}^{2}-\mu_{0}^{2}\right) F(\omega \theta) / \theta
$$

Relationship 4 can be rearranged to give an expression for the triplet state dipole moment in terms of the measured quantity $\left[\phi_{\text {isc }} \Delta M_{\mathrm{T}}\right]$.

$$
\mu_{\mathrm{T}}=\left\{\left[\phi_{\text {isc }} \Delta M_{\mathrm{T}}\right] \theta /\left(F(\omega \theta) K \phi_{\text {isc }}\right)+\mu_{0}{ }^{2}\right\}^{0.5}
$$

The ground state dipole moment of PNA is known to be $\mu_{0}$ $=6.2 \mathrm{D}^{13}$ In addition, we have determined the rotational charge mobility of the ground state, $M_{0}$, as given by (6), at the frequency

$$
M_{0}=K \mu_{0}^{2} F(\omega \theta) / \theta
$$

of our measurements to be $1.2 \times 10^{-8} \mathrm{~m}^{2} /(\mathrm{V} \mathrm{s})$. This allows us to derive a value for the dipole relaxation time for PNA in benzene of $29 \mathrm{ps}$. The only remaining unknown parameter in (5) is then the intersystem crossing efficiency.

Taking $\phi_{\text {isc }}$ to have its maximum possible value of 1 will result in the calculation of a minimum value of the triplet state dipole moment. For PNA in benzene this is $\mu_{\mathrm{T}}=11.3 \mathrm{D}$. An intersystem crossing efficiency for PNA close to unity in benzene is in fact probably reasonable in view the complete lack of fluorescence and the strong phosphorescence of this molecule in nonpolar media. If $\phi_{\text {isc }}$ were to be as low as 0.5 , $\mu_{\mathrm{T}}$ would be calculated to be $14.7 \mathrm{D}$. Since the dipole moment of the $S_{1}$ state has been determined to be approximately $15 \mathrm{D}$ on the basis of the electrochromism of the optical absorption, $2,3,12,13$ we conclude that the dipole moment of the $\mathrm{T}_{1}$ state is, almost certainly, lower than that of $S_{1}$.

Taking dipole relaxation to be controlled by rotational diffusion of the solute molecules with a characteristic time given by eq 7 ,we can estimate $\theta=62$ ps for PNA in dioxane on the

$$
\theta_{\mathrm{R}}=\eta V_{\mathrm{M}} / 6 k_{\mathrm{B}} T
$$

basis of the viscosity of $\eta=1.4 \mathrm{cP}$ compared with $\eta=0.65$ $\mathrm{cP}$ for benzene. In $7, V_{\mathrm{M}}$ is the volume of the solute molecule. Using $\theta=62 \mathrm{ps}$ in (5) together with the measured value of $\left[\phi_{\text {isc }} \Delta M_{\mathrm{T}}\right]$ for PNA in dioxane results in a value of $\mu_{\mathrm{T}}=8.8 \mathrm{D}$ for a quantum efficiency of 1 . This is much lower than the value of $11.3 \mathrm{D}$ calculated for benzene as solvent. This difference is possibly related to the considerable decrease in mixing between the lowest lying singlet and triplet states in dioxane as evidenced by the substantial increase in the $T_{1}$ lifetime, from 54 to $275 \mathrm{~ns}$. In benzene the strong mixing could result in a larger effective $\mathrm{T}_{1}$ state dipole moment compared with that of the "pure" ${ }^{3} \pi \pi^{*}$ state as a result of the $15 \mathrm{D}$ dipole moment associated with $\mathrm{S}_{1}$.

The reduced mixing in dioxane could also result in a lower intersystem crossing efficiency than in benzene. If $\phi_{\text {isc }}$ were in fact as low as 0.5 in dioxane, then a value of $\mu_{\mathrm{T}}=10.9 \mathrm{D}$ would be calculated which is close to the value of $11.3 \mathrm{D}$ found for benzene on the basis of $\phi_{\text {isc }}=1$.

The $\mathrm{T}_{1}$ dipole moments of the asymmetric $N$-alkyl derivatives DMPNA and 1-PNA can be estimated from their $\left[\phi_{\mathrm{isc}} \Delta M_{\mathrm{T}}\right]$ values using eq 5 and taking the dipole relaxation time to be proportional, to a first approximation, to the molecular volume (assumed proportional to the molecular weight) of the solute in 
TABLE 3: Triplet State Data from TRMC Transients

\begin{tabular}{lclllll}
\hline & & \multicolumn{4}{c}{$\mu_{\mathrm{T}}{ }^{c}(\mathrm{D})$} \\
\cline { 3 - 4 } \cline { 6 - 7 } & $\theta_{\mathrm{R}} / \eta$ & \multicolumn{2}{c}{$\phi_{\mathrm{isc}}=1$} & & \multicolumn{2}{c}{$\phi_{\mathrm{isc}}=0.5$} \\
\cline { 3 - 4 } \cline { 6 - 7 } solute & $(\mathrm{ps} / \mathrm{cP})$ & benzene & dioxane & & benzene & dioxane \\
\hline PNA & $45^{a}$ & 11.3 & 8.8 & & 14.7 & 10.9 \\
DMPNA & $54^{b}$ & 9.1 & 9.1 & & 11.1 & 11.1 \\
1-PNA & $67^{b}$ & 9.1 & 8.7 & & 11.0 & 10.5 \\
2-PNA & $107^{b}$ & $8.4(15.0)^{d}$ & $9.0(15.6)^{d}$ & & $11.8(18.4)^{d}$ & $12.7(19.3)^{d}$ \\
3-PNA & $148^{b}$ & $8.6(15.2)^{d}$ & $9.9(16.5)^{d}$ & & $12.2(18.8)^{d}$ & $14.0(20.6)^{d}$
\end{tabular}

${ }^{a}$ From measured $M_{0}=1.2 \times 10^{-8} \mathrm{~m}^{2} /(\mathrm{V}$ s $)$ and $\mu_{0}=6.2 \mathrm{D}$. ${ }^{b}$ Estimated from the value for PNA based on a first power dependence on molecular weight. ${ }^{c}$ The ground state dipole moments used were 6.2 (PNA), 6.6 (DMPNA and 1-PNA), and 0 D (2-PNA and 3-PNA). ${ }^{d}$ The estimated dipole moment of the locally excited moiety, taking for the opposing dipole moment of the dormant moieties a value of $6.6 \mathrm{D}$.

accordance with eq 7. The values of the viscosity normalized rotational diffusion time, $\theta_{\mathrm{R}} / \eta$, are listed in Table 3 . These values were used, together with a value of $6.6 \mathrm{D}$ for $\mu_{0},{ }^{13}$ to derive the $\mu_{\mathrm{T}}$ values for DMPNA and 1-PNA given in Table 3. The values are seen to be very similar for both compounds and solvent independent with average values of 9.0 and 11.0 D for $\phi_{\text {isc }}=1$ and 0.5 , respectively. Interestingly these values are also close to those of 8.8 and 10.9 D found for PNA in dioxane.

We have been unable to find measured values of $\phi_{\text {isc }}$ for the present compounds in the literature. The 2:1 ratio found for the phosphorescence to fluorescence yields for DMPNA at low temperatures would suggest however that $\phi_{\text {isc }}$ is probably considerably less than unity and possibly closer to 0.5 than to 1. We conclude that the dipole moments of the pure ${ }^{3} \pi \pi^{*}$ states of PNA and its $N$-alkyl substituted derivatives are probably closer to $11 \mathrm{D}$ than to the lower limit of $9 \mathrm{D}$. The former value is similar to the value of $12 \mathrm{D}$ recently estimated for the dipole moment of the triplet state of (dimethylamino)benzonitrile using the present technique. ${ }^{22}$

The large TRMC transients observed for the symmetrical compounds 2-PNA and 3-PNA indicate that the excitation energy must remain localized on a single nitro-anilino moiety for a time at least comparable with and possibly considerably longer than the $c a$. 15 ps reciprocal radian frequency of the microwaves. Taking initially the dipole relaxation times to be controlled by rotational diffusion and given by (7), we have calculated the net dipole moments of the excited states with a value of zero for the ground state dipole moment, i.e., using

$$
\mu_{\mathrm{T}}=\left\{\left[\phi_{\text {isc }} \Delta M_{\mathrm{T}}\right] \theta /\left(F(\omega \theta) K \phi_{\text {isc }}\right)\right\}^{0.5}
$$

The values of $\mu_{\mathrm{T}}$ obtained are given in Table 3 and are seen to be of the same order of magnitude or even larger than those determined for the nonsymmetrical DMPNA and 1-PNA compounds.

There is in fact a larger discrepancy between the dipole moment values for the symmetrical and nonsymmetrical compounds than might appear at first sight from the calculated values of $\mu_{\mathrm{T}}$ in Table 3 . This arises because one has to take into account that $\mu_{\mathrm{T}}$ for the symmetrical compounds is a net value, equal to the resultant of the dipole associated with the locally excited chromophore, $\mu_{\mathrm{LT}}$, and the opposing dipole(s) of the accompanying "dormant" nitro-anilino moiety(ies). If the latter is taken as a first approximation to be close to the value of 6.6 $\mathrm{D}$ found for ground state DMPNA, then the dipole moment of the locally excited chromophore must be larger than the $\mu_{\mathrm{T}}$ values given in Table 3 by approximately this amount. These estimates of $\mu_{\mathrm{LT}}$ are the values shown in parentheses in Table
3. Even when a maximum quantum yield of unity is used, unrealistically large values, in excess of $15 \mathrm{D}$, are found.

It would appear that the values of the dipole relaxation times used to derive the $\mu_{\mathrm{T}}$ values using eq 8 for the symmetrical compounds are too large. It is however very unlikely that the rotational relaxation times of 2- and 3-PNA are smaller than the values determined using (7) since this relationship assumes a spherical molecular geometry. The more cylindrical and diskshaped actual geometries of these molecules would result, if anything, in the calculation of even larger values of $\theta_{\mathrm{R}}$ than those based on a spherical approximation. ${ }^{20}$ This would in turn result in even larger estimates of $\mu_{\mathrm{T}}$.

We are forced to conclude that dipole relaxation in the triplet state of 2- and 3-PNA occurs more rapidly than rotational relaxation and therefore that there is an additional mechanism which causes dipole reversal. This most probably involves flipflop switching of the local dipole between the different possible degenerate locally excited structures, as has been proposed to occur for a similar symmetrical derivative of (dimethylamino)benzonitrile. ${ }^{23,24}$ Such a flip-flop mechanism would be expected to be only weakly, if at all, dependent on solvent viscosity. This may explain why the values calculated on the basis of rotational relaxation times are consistently larger for the more viscous solvent, dioxane. We conclude finally therefore that the time scale for flip-flop dipole relaxation in the $T_{1}$ states of the symmetrical compounds must be comparable with or shorter than the rotational relaxation times given in Table 3 , in other words probably on the order of several tens of picoseconds.

\section{References and Notes}

(1) Moll, F.; Lippert, E. Z. Elektrochem. 1954, 58, 853.

(2) Czekalla, J.; Wick, G. Z. Elektrochem. 1961, 65, 727.

(3) Liptay, W.; Eberlein, W.; Weidenberg, H.; Elflein, O. Ber. BunsenGes. Phys. Chem. 1967, 71, 548.

(4) Seliskar, C.; Khalil, O.; McGlynn, S. In Excited States; Lim, E. C., Ed.; Academic Press: New York, 1974; Vol. I, p 279-288.

(5) Wolleben, J.; Testa, A. C. J. Phys. Chem. 1977, 81, 429.

(6) Bertinelli, F.; Palmieri, P.; Brillante, A.; Taliani, C. Chem. Phys. 1977, 25, 333 .

(7) Kanno, H.; Terazima, M.; Azumi, T. J. Phys. Chem. 1991, 95, 3498.

(8) Carsey, T. P.; Findley, G. L.; McGlynn, S. P. J. Am. Chem. Soc. 1979, 101, 4502.

(9) Khalil, O. S.; Seliskar, C. J.; McGlynn, S. P. J. Chem. Phys. 1973, $58,1607$.

(10) Terazima, M.; Kanno, H.; Azumi, T. Chem. Phys. Lett. 1990, 173, 327.

(11) Kallir, A. J.; Suter, G. W.; Wild, U. P. J. Phys. Chem. 1987, 91, 60.

(12) Liptay, W. In Excited States; Lim, E. C., Ed.; Academic Press: New York, 1974; Vol. I, p 197.

(13) McClellan, A. L. Tables of Experimental Dipole Moments; Rahara Enterprises: El Cerrito, CA, 1974 (Vol. 2); 1989 (Vol. 3).

(14) Ledoux, I.; Zyss, J.; Siegel, J. S.; Brienne, J.; Lehn, J.-M. Chem. Phys. Lett. 1990, 172, 440-444.

(15) Joffre, M.; Yaron, D.; Silbey, R. J.; Zyss, J. J. Chem. Phys. 1992, 97, 5607-5615.

(16) Brédas, J.-L.; Meyers, F.; Pierce, B. M.; Zyss, J. J. Am. Chem. Soc. 1992, 114, 4928.

(17) Zyss, J. J. Chem. Phys. 1993, 98, 6583-6599.

(18) Zyss, J.; Dhenaut, C.; Chauvan, T.; Ledoux, I. Chem. Phys. Lett. 1993, 206, 409-414.

(19) de Haas, M. P.; Warman, J. M. Chem. Phys. 1982, 73, 35.

(20) Schuddeboom, W. Ph.D. Thesis (Delft University of Technology: Delft, The Netherlands, 1994; ISBN 90-73861-21-7).

(21) The $T_{1}$ lifetime of PNA decreases with increasing solute concentration. The value quoted was obtained by extrapolation to zero concentration. This self-quenching effect, which was specific for PNA, will be the subject of a separate publication.

(22) Schuddeboom, W.; Jonker, S. A.; Warman, J. M.; Leinhos, U.; Kühnle, W.; Zachariasse, K. A. J. Phys. Chem. 1992, 96, 10809.

(23) Jonker, S. A.; Warman, J. M. Chem. Phys. Lett. 1991, 185, 36.

(24) Warman, J. M.; Schuddeboom, W.; Jonker, S. A.; de Haas, M. P.; Paddon-Row, M. N.; Zachariasse, K. A.; Launay, J.-P. Chem. Phys. Lett. 1993, 210, 397.

JP9606826 